\title{
Industrie 4.0 in kleinen und mittleren Unternehmen - Welche Potenziale lassen sich mit smarten Geräten in der Produktion heben?
}

\author{
Ulrich Matthias König Projektgruppe Wirtschaftsinformatik des Fraunhofer FIT, Kernkompetenzzentrum \\ Finanz- \& Informationsmanagement, Universität Augsburg \\ Universitätsstraße 12, 86159 Augsburg, ulrich.koenig@ fim-rc.de, 0821 / 598 -4844
}

\begin{abstract}
Maximilian Röglinger Projektgruppe Wirtschaftsinformatik des Fraunhofer FIT, Kernkompetenzzentrum
\end{abstract} Finanz- \& Informationsmanagement, Universität Bayreuth

\author{
Nils Urbach
}

Projektgruppe Wirtschaftsinformatik des Fraunhofer FIT, Kernkompetenzzentrum Finanz- \& Informationsmanagement, Universität Bayreuth

This is a post-peer-review, pre-copyedit version of an article published in HMD - Praxis der

Wirtschaftsinformatik. The final authenticated version is available online at: http://dx.doi.org/ 10.1365/s40702-019-00567-w

\begin{abstract}
Zusammenfassung
Die hohe Anzahl genutzter smarter Geräte führt zu deren weiter Verbreitung und engen Integration im Alltag. Mit der Erweiterung von Alltagsgegenständen um Netzwerkkonnektivität, dem Internet der Dinge (Internet of Things, IoT), ist ein neuer Trend beobachtbar. Das Internet der Dinge bietet zahlreiche Einsatzgebiete und katalysiert die Verschmelzung von physischer und digitaler Welt. Dadurch lassen sich insbesondere Kommunikation und Interaktion zwischen Individuen, Gegenständen und Unternehmen verbessern. In der Industrie muss zur Integration und Potenzialnutzung des Internets der Dinge der Kontext gewissenhaft analysiert werden. Plant ein Unternehmen eine Transformation hin zu Industrie 4.0, so muss es Abhängigkeiten zu Produktionsanlagen und Anwendungssystemen berücksichtigen. Motiviert durch Effizienzpotenziale, haben große Unternehmen bereits mit der Transformation begonnen. In kleinen und mittleren Unternehmen (KMU) wird die Umstellung oft noch defensiv betrachtet. Jedoch bietet sich auch für KMU großes Kostenreduktions- und Prozessverbesserungspotenzial. Diese Problemstellung adressiert das von der Bayerischen Forschungsstiftung geförderte Forschungsprojekt ,,SmarDes@Work - Smart Devices in der Produktion “. Ziel war es, handelsübliche smarte Geräte einfach in die Produktionsprozesse von KMU zu integrieren. Im Rahmen des Forschungsprojekts erarbeitete ein Konsortium aus Wissenschaftlern, Produktionsbetrieben und Softwareherstellern eine Startlösung für Industrie 4.0 in KMU. In diesem Beitrag werden die zentralen Erkenntnisse vorgestellt und Handlungsempfehlungen abgeleitet.
\end{abstract}

Schlüsselwörter: Smarte Geräte, Produktion, Prozessverbesserung, KMU, Industrie 4.0, Internet der Dinge

\begin{abstract}
The growing number of smart devices leads to widespread use and close integration to daily life. A new trend, the Internet of Things (IoT), has emerged as a result of technology being closely integrated with everyday objects. The IoT offers numerous areas of application and catalysis the fusion of the physical and digital worlds. In this way, communication and interaction between individuals, objects, and organizations can be improved. In manufacturing, the integration and exploitation of the potential of IoT requires a careful analysis of the context. If a company plans for a transformation to industry 4.0, they must consider dependencies on production facilities and application systems. Motivated by efficiency potentials, large companies have already started the transformation. In small and medium-sized enterprises (SMEs), the changeover is often still viewed defensively. However, there is also a great potential for cost reduction and process improvement for SMEs. This problem is addressed by the research project "SmarDes@Work - Smart Devices in Production" funded by the Bavarian Research Foundation. The aim of the research project was to easily integrate commercially available smart devices into the production processes of SMEs. As part of this research project, a consortium of academics, production companies, and software developers developed a starting solution for Industry 4.0 in SMEs. In this paper, the central findings are presented and recommendations for action are derived.
\end{abstract}

Key words: Smart Device, Production, Process improvement, SME, Industry 4.0, Internet of Things 


\section{Motivation}

Der Start der Digitalisierung mittels digitaler Technologien für Privatpersonen begann mit der Einführung des Smartphones und entwickelte sich zu einem essentiellen Bestandteil des alltäglichen Lebens (Fleisch et al. 2015). Kurze Entwicklungszyklen, steigende Gerätezahlen und deren kostengünstige Anschaffung sind Gründe für die stark steigende Nutzung von smarten Geräten im privaten Umfeld (Püschel et al. 2017). Eine aufkommende Technologie ist das Internet der Dinge (Internet of Things, IoT). Bei IoT handelt es sich um physische Gegenstände, die mit Sensoren, Aktuatoren, Rechenleistung und Netzwerkkonnektivität ausgestattet werden (Oberländer et al. 2018; Porter und Heppelmann 2014). Diese Gegenstände sind durch die Möglichkeit der starken Vernetzung ein Treiber für die Verschmelzung von digitaler und physischer Welt (Püschel et al. 2017). Beispielhafte Anwendungsdomänen sind die Bereiche Smart Home, Gesundheitswesen, Mobilität oder persönliches Wohlbefinden (Oberländer et al. 2018). Aktuellen Studien zur Folge stagniert die Entwicklung von smarten Geräten nicht. Im Gegenteil: Bis zum Jahr 2030 sollen weltweit bis zu 500 Milliarden smarte Geräte auf dem Markt sein und zu einem Umsatz von bis zu 267 Milliarden US-Dollar im Jahr 2020 führen (Muccini und Moghaddam 2018).

Im Jahr 2011 wurde die vierte industrielle Revolution oder „Industrie 4.0“-Initiative in Deutschland als Antwort auf die Digitalisierung im Industrieumfeld initiiert (Kagermann et al. 2011). Ein Teil dieser Entwicklung ist die Steigerung der Produktivität, Flexibilität und Individualisierung der Produktion durch digitale Technologien. Ein Lösungsansatz ist die Nutzung bereits vorhandener Gegenstände, erweitert um Sensorik, Rechenleistung und Netzwerkkonnektivität (Rüßmann et al. 2015). Entscheidend hierbei ist, wie IoT die Transformation zu Industrie 4.0 gestalten kann. Große Unternehmen, insbesondere aus der Automobilindustrie, berichten bereits über erste Erfolge (BMW Group 2015; Virtual Reality Magazin 2015). Im Vergleich zu Privathaushalten ist die Integration von IoT in Unternehmen oft nicht niedrigschwellig und kostengünstig realisierbar. Es müssen Abhängigkeiten zu vorhandenen, meist heterogenen Produktionsmaschinen, Anwendungssystemen und Netzwerkinfrastrukturen berücksichtigt werden. Für eine leichtgewichtige Nutzung von IoT in der Produktion fehlt es an einer standarisierten Integrationsplattform, um Produktionsmaschinen, Anwendungssysteme, menschliche Akteure und smarte Geräte zu verbinden.

Fehlende Standards und überdimensionierte Lösungen sind ein Grund, weshalb sich kleine und mittlere Unternehmen (KMU) aktuell nur defensiv in Richtung Industrie 4.0 entwickeln (Albayrak und Gadatsch 2018). Laut dem Statistischem Bundesamt (2019) gehören 99,3\% der deutschen Unternehmen zu den KMU, die international für ihre hervorragenden Leistungen bekannt sind. Daraus lässt sich ein hohes Potenzial aus der Integration von IoT für produzierende KMU ableiten. Aus diesem Grund förderte die Bayerische Forschungsstiftung im Rahmen des Forschungsprojekts „SmarDes@Work-Smart Devices in der Produktion“die Entwicklung einer Software-Integrationsschicht für KMU. Das Projektkonsortium entschied sich für die Nutzung handelsüblicher smarter Geräte, wie Smartphones, Tablets und smarter Uhren. Die Hauptgründe waren deren hohe Verbreitung, intuitive Nutzung sowie geringe Anschaffungskosten. Im Verlauf des Forschungsprojekts zeigte sich, dass die Mehrheit der Beschäftigten von KMU bereits im Umgang mit smarten Geräten, insbesondere Smartphones, geübt und somit vertraut sind mit der Nutzung von Client-Applikationen. Ein weiterer Vorteil für KMU ist die kostengünstige Anschaffung handelsüblicher smarter Geräten und deren Austauschbarkeit bei Defekten. Auf Basis unserer Projektergebnisse wird in diesem Beitrag die folgende Forschungsfrage beantwortet: Wie lassen sich smarte Geräte nutzenstiftend in produktionsnahe Prozesse von KMU integrieren?

Anhand von acht Anwendungsfällen entwickelte und evaluierte ein Konsortium, bestehend aus zwei wissenschaftlichen, vier industriellen und zwei softwarenahen Partnern, als Startlösung eine Integrationslösung mit zugehöriger Client-Applikation für ausgewählte smarte Gerätetypen. Bevor drei Anwendungsfälle, die exemplarisch wie auch allumfassend die Funktionalitäten der Integrationslösung beschreiben, und deren Ergebnisse vorgestellt werden, skizzieren wir Ausgangssituation, grundlegend alle Anwendungsfälle und den Lösungsansatz. Auf Basis der Ergebnisse leiten sich allgemeine Handlungsempfehlungen und übergreifende Erkenntnisse ab. 


\section{Ausgangssituation - Industrie 4.0 in KMU}

Insbesondere KMU stellt die Digitalisierung aufgrund von Kostendruck und limitierter Ressourcen vor die Herausforderung einer zunehmenden Variantenvielfalt, Flexibilität und Individualisierung von Produkten und Prozessen (Albayrak und Gadatsch 2018). Ein Ansatz, diese Herausforderungen zu adressieren, ist die Realisierung von Prozessverbesserungen durch die nahtlose Integration digitaler Technologien. Dies führt zu einer Verschmelzung von digitaler und physischer Welt und zu teils komplexen Plattformlösungen (Püschel et al. 2017). Limitierte Verfügbarkeit von Ressourcen, sowie teilweise fehlendes Fachwissen im Bereich Digitalisierung, sind für KMU weitere Herausforderungen (Albayrak und Gadatsch 2018). Vor allem begrenzte Mitarbeiterressourcen führen zu einem zögerlichen Vorgehen, da bestehende Produktionsmaschinen und produktionsnahe Anwendungssysteme bei laufendem Betrieb erweitert werden müssen - und oftmals weder aus inhaltlichen noch finanziellen Gründen ersetzt werden können.

Produktionsrelevante Anwendungssysteme sind Enterprise-Resource-Planning-Systeme (ERP-Systeme), Betriebsdatenerfassung (BDE) und Manufacturing-Execution-Systeme (MES) (Hausladen 2010; Winkelmann und Klose 2008). Diese Systeme stammen meist von unterschiedlichen Anbietern, sind Eigenentwicklungen oder haben sich durch intensives Customizing über die Zeit zu „Individuallösungen“ entwickelt (Winkelmann und Klose 2008). Unserer Erfahrung nach nutzen gerade kleine Unternehmen keine etablierten Systeme, sondern steuern die Produktion auf Basis von Tabellenkalkulationsprogrammen. Des Weiteren werden zur Integration neuer Technologien, wie smarte Geräte oder Cloud-Computing, Anbieterwechsel inklusive Datentransformation benötigt, die in der Regel mit großen Herausforderungen für KMU verbunden sind (Leyh und Bley 2016). Abschließend konzentrieren sich bestehende Lösungen meist auf die Verbesserung einzelner Prozessschritte und lassen somit eine Ende-zu-Ende-Perspektive auf Geschäftsprozesse vermissen (Hausladen 2010).

Im Rahmen von Industrie 4.0 müssen KMU allerdings umfassend auf die Vernetzung von Mitarbeitern, Produktionsmaschinen, Anwendungssystemen und Produkten setzen (Bertschek et al. 2015). Durch vertikale und horizontale Vernetzung lässt sich IoT nutzenstiftend integrieren, sodass nahezu eine Echtzeitkommunikation ermöglicht wird und Produktionsprozesse flexibler werden (Arbeitskreis Industrie 4.0 2013). Zentral sind zudem eine fundierte Auswahl und Analyse verfügbarer Technologien und Geräte. Unter Kosten- und Effizienzgesichtspunkten sollten nicht nur komplexe Plattformlösungen und moderne cyber-physische Produktionsmaschinen verwendet werden, sondern ebenso leichtgewichtige und kostengünstige Ansätze. Unter Berücksichtigung des Kostenaspekts scheinen handelsübliche smarte Geräte, wie Smartphones, Tablets oder smarte Uhren, hierfür geeignet zu sein. Smarte Geräte sind physische Gegenstände, die kabellos und mobil sind, sowie über Sensoren und Aktoren verfügen (Fraunhofer IML 2019). Handelsübliche smarte Geräte erfreuen sich zudem einer hohen Verbreitung, sodass für KMU der Schulungsaufwand und etwaiger Widerstand bei der Einführung überschaubar scheint (Püschel et al. 2017). Zudem berichten Unternehmen aus der Automobilbranche bereits über erste Erfahrung bei der Nutzung smarter Geräten in der Produktion (BMW Group 2015; Virtual Reality Magazin 2015). Aus diesem Grund schien es zu Beginn des Forschungsprojekts „SmarDes@Work - Smart Devices in der Produktion“ diese spezielle Art des IoT als geeignet für die Realisierung von integrierten Prozessverbesserungen in KMU.

Als Intermediär zwischen Maschinen, Produktionsmitarbeitern und Anwendungssystemen ist es denkbar, smarte Geräte bei der Arbeitsvorbereitung und Intralogistik wie auch in der Produktion selbst einzusetzen. Zudem können smarte Geräte die Kommunikation in der Produktion beschleunigen. Dadurch können Ressourcen effizienter genutzt, kontextspezifische Informationen zeitnah bereitgestellt und eine unmittelbarere Steuerung der Produktionsabläufe erreicht werden. Somit scheint es sinnvoll, KMU leichtgewichtige und niedrigschwellige Lösungen anzubieten, um eine schrittweise Integration von IoT sowie eine Transformation hin zu Industrie 4.0 zu ermöglichen. Ebenso sollte die frühzeitige und zeitnahe Nutzenrealisierung einer Lösung angestrebt werden. 


\section{Projektvorgehen}

Zur Adressierung der skizzierten Herausforderungen von KMU mittels smarter Geräte eignete sich der Ansatz der Konsortialforschung. Dieser Ansatz ist durch die frühe Integration von potentiellen Nutzern charakterisiert, sodass bereits zum Zeitpunkt der Ausarbeitung Rückmeldungen direkt von Unternehmen erhalten werden (Österle und Otto 2010). Im Rahmen des durch die Bayerische Forschungsstiftung geförderten Forschungsprojekts „SmarDes@Work - Smart Devices in der Produktion“ entstand die Möglichkeit, in einem realweltlichen Umfeld Demonstratoren für eine KMU-taugliche industrielle Integrationslösung zu entwickeln und zu evaluieren. Konkret bestand das Ziel des Forschungsprojekts darin, einen Demonstrator für eine Middleware als Integrationsschicht und Client-Applikationen zur Vernetzung von Produktionsmaschinen, Produktionsmitarbeitern, Anwendungssystemen und smarten Geräten zu entwickeln. Das Konsortium entschloss sich zu einem vierphasigen Vorgehen (siehe Abbildung 1).

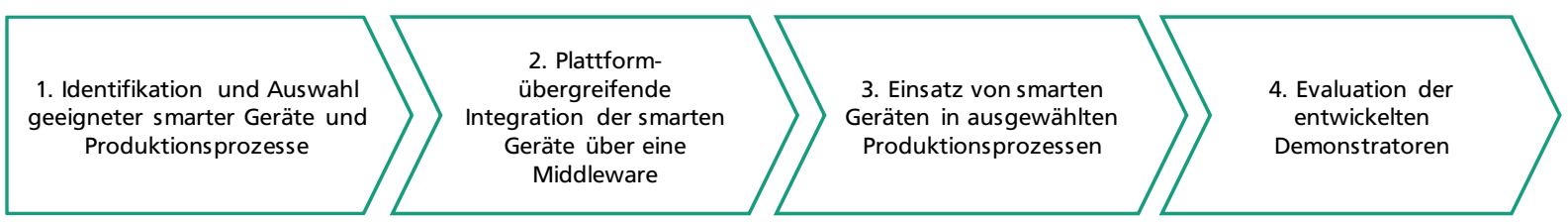

Abbildung 1: Vorgehen im Forschungsprojekt anhand von vier Phasen

In Phase 1 bestand das Ziel in der Identifikation möglicher Produktionsbereiche und konkreter Produktionsprozesse mit Verbesserungspotenzial. Zudem erfolgte eine Marktanalyse smarter Geräte, sodass eine geeignete Auswahl für die identifizierten Anwendungsfälle erfolgen konnte. Als Kernergebnis dieser Phase standen acht ausführlich beschriebene Anwendungsfälle und die Entscheidung, im Rahmen des Forschungsprojekts den Fokus auf die Gerättypen Smartphones, Tablets, smarte Uhren sowie Fitnessarmbänder zu setzen. Die Auswahl dieser Gerätetypen erfolgte anhand einer detaillierten Analyse der Anwendungsfälle, Interviews mit beteiligten Personen sowie der Gegenüberstellung der Ziele der Anwendungsfälle und Funktionalitäten der smarten Geräte.

Im Fokus von Phase 2 standen die Konzeption sowie Implementierung der Demonstratoren. Das Konsortium entschied sich zur Entwicklung einer Middleware, die softwarebasierte Dienste über standardisierte Schnittstellen zur Verfügung stellt. Die Entwicklung einer Middleware schien sinnvoll, da es sich um ein etabliertes Konzept des Enterprise-Architektur-Managements handelt, um die Kommunikation verschiedener Systeme standardisiert sicherzustellen (Aier und Schelp 2008). Des Weiteren stellt eine modulare Architektur der Middleware eine breite Anwendbarkeit und Erweiterungsfähigkeit sicher. Vor der agilen Entwicklung standen allerdings das initiale Erfassen und Strukturieren von Anforderungen. Während der Definition der Middleware-Dienste wurde früh vom Konsortium bestätigt, dass eine zentrale Anforderung die nahtlose Kommunikation zwischen Komponenten und Mitarbeitern sei. Dies führte zur Entscheidung, eine nachrichtenbasierte Middleware-Lösung anzustreben.

Phase 3 ergänzte den Funktionsumfang der Middleware und umfasste daher die Definition und Umsetzung funktionaler und nichtfunktionaler Anforderungen an die Client-Applikation und die smarten Geräte, um die einwandfreie Kommunikation beider Demonstratoren zu gewährleisten. Aus den Anforderungen wurden zudem Maßnahmen zum Schutz der smarten Geräte abgeleitet, bewertet und getestet. Dies war notwendig, da handelsübliche smarte Geräte nicht standardmäßig für besondere Begebenheiten in der Produktion, z.B. Stürze und Kontakt mit Betriebsmitteln, vorbereitet sind. Nach den Vorarbeiten der ersten Phasen war es in Phase 3 möglich, erste Tests der Software-Demonstratoren und smarten Geräte durchzuführen. Mit diesem Vorgehen erhielten alle Partner frühzeitig Rückmeldung über fehlende Funktionalitäten, die bis Projektende berücksichtigt wurden.

Im Vordergrund von Phase 4 stand die iterative Evaluation der Demonstratoren und der smarten Geräte. In mehreren mehrwöchigen Testläufen wurden sequenziell pro Anwendungspartner die Anwendungsfälle im jeweiligen Unternehmen integriert und von den Produktionsmitarbeitern getestet. Zum Ende der Testphasen wurden die zentralen Ergebnisse und Erkenntnisse im Rahmen von semi-strukturierten Interviews erfasst, ausgewertet und kommuniziert. Durch dieses Vorgehen gelang es, eine Lösung zu entwickeln, welcher ein erheblicher Mehrwert und Kommunikationsverbesserungen zugesprochen wurden. 


\section{Lösungsansatz}

Auf Basis der vorgestellten Projektkonstellation war es möglich, während des Projekts acht Anwendungsfälle in mehreren Iterationen realweltlich zu evaluieren. Zur besseren Nachvollziehbarkeit der Komponenten ist in Abbildung 2 der generische Aufbau der Lösung mit möglichen Produktionsmaschinen, Anwendungssystemen und Produktionsmitarbeitern schematisch dargestellt. Den Kern bilden die Middleware und die durch die smarten Geräte repräsentierte Client-Applikation. Dieser Kern verbessert die Mensch-Maschine-Interaktion der an der Produktion beteiligten Mitarbeiter in produktionsnahen Prozessen. Vereinfachend seien hier die Rollen Produktionsmitarbeiter und Schichtleiter genannt, die über die Middleware und smarte Geräte als Kommunikationsintermediäre entweder Nachrichten aus Anwendungssystemen oder von Produktionsmaschinen erhalten bzw., je nach Anwendungsfall, auch untereinander Nachrichten austauschen können.

Die Middleware wird dabei in die bestehende Anwendungslandschaft der Unternehmen integriert und auf einem Server installiert. Dabei ist es aufgrund der generischen Implementierung irrelevant, ob die Middleware auf einem bereits vorhandenen System oder auf einem zusätzlichen installiert wird. Entscheidend ist lediglich, dass vom Server der Zugriff auf die für den Anwendungsfall relevanten Anwendungssysteme und das Internet sichergestellt ist. Die Client-Applikation wird entsprechend auf den smarten Geräten installiert. Hierbei werden eine Internetund interne Netzwerkkonnektivität benötigt. Die allgemeine Internetkonnektivität wird für das Versenden der Notifikationen von Middleware zur Client-Applikation benötigt.

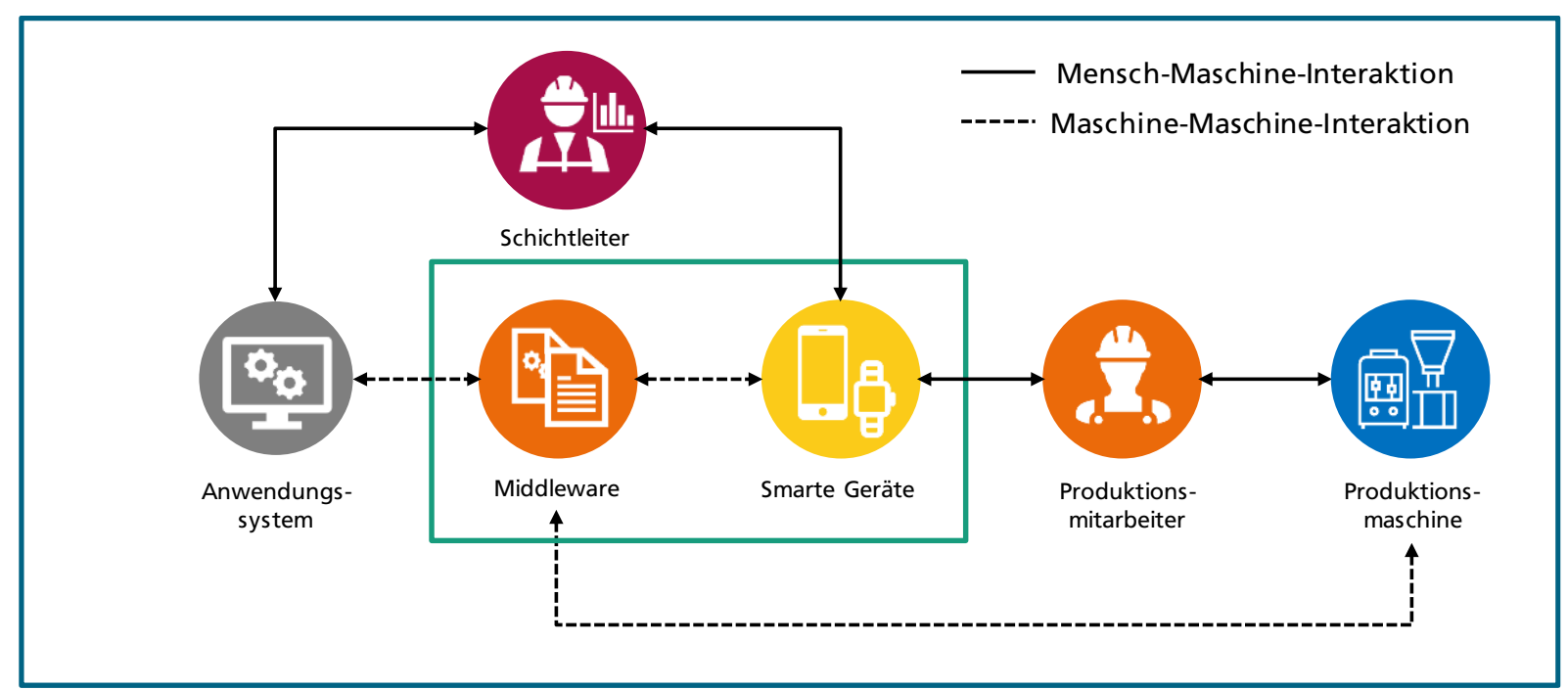

Abbildung 2: Gesamtüberblick über die Komponenten des Forschungsprojekts

Die nachrichtenbasierte Middleware erhält zur Initiierung eines Vorgangs einen Trigger. Ein Trigger kann von Produktionsmaschinen, Anwendungssystemen oder einem Produktionsmitarbeiter mittels smartem Gerät ausgelöst werden. Nach der Initiierung erfolgen eine Filterung und eine Auswertung anhand verschiedener Kriterien, bevor die Nachricht weitergeleitet wird. Mehrere Nachrichten werden, von der Middleware gesteuert, in Konversationen zusammengefasst. Dies erfolgt über den zentral verwalteten Dienst der Middleware, den Message Broker auf Basis von Active MQ Artemis. Für den Empfang von Maschinendaten besteht eine Schnittstelle, die GatewayKomponente (PLC-Integration), die auf der einen Seite an die Maschinensteuerung angebunden ist und auf der anderen Seite die Nachrichtendaten an den Message Broker weiterleitet. Zur Konfiguration des Message Brokers wurden eine Regelsprache und eine Rule Engine spezifiziert. Mit Hilfe der Rule Engine war es möglich, Middleware und Client-Applikation auf XML-Basis einfach für die Anwendung in Unternehmen zu konfigurieren.

Ergänzend zur Middleware entstand als weiteres zentrales Projektergebnis eine Client-Applikation, die sich in zwei Komponenten aufteilt. Das Smart Devices Gateway stellt die Kommunikation der smarten Geräte mit der Middleware sicher. Die zweite Komponente ist die Client-Applikation selbst, die eine geeignete Darstellung der Daten sowie Interaktionen ermöglicht. Analog zur Middleware ist die Client-Applikation ebenfalls flexibel über 
vier Sichten konfigurierbar. In der Echtzeitsicht ist die Anzeige von Produktionsdaten möglich. Dabei sind Diagramme zur Visualisierung von Werten vorhanden. Die Dashboard-Sicht ermöglicht es, Interaktion über Buttons zu definieren, zum einen als Reaktion auf eine Nachricht oder als Trigger. In der Nachrichtensicht werden aktuelle von der Middleware weitergeleiten Nachrichten angezeigt. In der Job-Sicht sind abschließend konkrete Handlungen visualisiert, die zur Reaktion auf Nachrichten definiert sind.

Die Implementierung erfolgte OpenSource, sodass nach Abschluss des Projekts die Ergebnisse öffentlich nutzbar sind. Aufgrund des agilen Vorgehens im Forschungsprojekt war es möglich, jeden Anwendungsfall mindestens zweimal zu evaluieren.

\section{Anwendungsfälle}

Zur Veranschaulichung des Funktionsumfangs der Demonstratoren und der daraus resultierenden Handlungsempfehlungen für die Integration von smarten Geräten in die Produktionsprozesse von KMU werden in Tabelle 1 die acht im Forschungsprojekt realisierten Anwendungsfälle präsentiert. Aus der Gesamtmenge werden im Folgenden drei repräsentative Anwendungsfälle herausgegriffen und beschrieben. Bei den drei ausgewählten Anwendungsfällen handelt es um jene, die in ihrer Gesamtbetrachtung umfassend die Funktionalitäten der Demonstratoren beschreiben.

Tabelle 1: Kurzübersicht der acht Anwendungsfälle

\begin{tabular}{|c|c|}
\hline Titel & Kurzbeschreibung \\
\hline $\begin{array}{l}\text { Werkzeuginstandsetzung } \\
\text { bei Maschinenausfällen }\end{array}$ & $\begin{array}{l}\text { Der Produktionsmitarbeiter signalisiert über ein smartes Gerät einem Logistiker } \\
\text { den Transport eines Werkzeugs in die Instandhaltung. Der Instandhalter wird } \\
\text { durch das smarte Gerät über das bevorstehende Eintreffen informiert. }\end{array}$ \\
\hline $\begin{array}{l}\text { Dokumentation von } \\
\text { Maschinenstillständen } \\
\text { und Fehlererfassung }\end{array}$ & $\begin{array}{l}\text { Bei Maschinenstopp muss der Grund für den Stillstand in einem entfernten BDE- } \\
\text { Terminal eingegeben werden. Mittels eines smarten Gerätes soll die Eingabe } \\
\text { direkt an der Maschine erfolgen, um so die Datenqualität zu erhöhen. }\end{array}$ \\
\hline $\begin{array}{l}\text { Anleitung für Maschinen- } \\
\text { einstellungen }\end{array}$ & $\begin{array}{l}\text { Der Produktionsmitarbeiter erhält eine Anleitung mit den wichtigsten Elementen, } \\
\text { welche beim Einrichten oder Warten von Produktionsmaschinen zu berücksich- } \\
\text { tigen sind. }\end{array}$ \\
\hline $\begin{array}{l}\text { Bearbeitung von } \\
\text { Materialanfragen }\end{array}$ & $\begin{array}{l}\text { Materialanfragen sollen dem Produktionsmitarbeiter mittels Benachrichtigung } \\
\text { auf einem smarten Gerät mitgeteilt werden. Zudem erfolgt eine Warnung, falls } \\
\text { nicht vollständig vorbereitetes Material droht, in die Produktion einzugehen. }\end{array}$ \\
\hline $\begin{array}{l}\text { Unterstützung von } \\
\text { Rüstprozessen mithilfe } \\
\text { von Echtzeitdaten }\end{array}$ & $\begin{array}{l}\text { Die Anzahl gefertigter Teile soll in Echtzeit erfasst, im ERP-System dokumen- } \\
\text { tiert und auf dem smarten Gerät ausgegeben werden. Es wird kalkuliert, wann die } \\
\text { Rüstung begonnen werden muss und der Logistiker erhält zeitnah eine Nachricht. }\end{array}$ \\
\hline $\begin{array}{l}\text { Anleitung für } \\
\text { Werkzeugwartung }\end{array}$ & $\begin{array}{l}\text { Bei Ausfall eines erfahrenen Produktionsmitarbeiters fehlt die Kompetenz, ein } \\
\text { Werkzeug sicher zu warten. Um diese Problematik zu vermeiden, wird eine An- } \\
\text { leitung auf dem smarten Gerät angezeigt, die schrittweise durchgegangen wird. }\end{array}$ \\
\hline $\begin{array}{l}\text { Benachrichtigung bei } \\
\text { vorausschauender } \\
\text { Fehlererkennung }\end{array}$ & $\begin{array}{l}\text { Die Maschinen bzw. das MES überwachen Sensoren einer Produktionsanlage } \\
\text { und melden Fehler an die Middleware. Die aufgetretene Störung wird je nach } \\
\text { Ursprung an ein smartes Gerät gesendet. }\end{array}$ \\
\hline $\begin{array}{l}\text { Bedarfsgerechte } \\
\text { Aufgabenerinnerung }\end{array}$ & $\begin{array}{l}\text { Mitarbeiter werden bei einem niedrigen Materialfüllstand über smarte Geräte in- } \\
\text { formiert. Verschiedene Signale werden verwendet, um diverse Materialfüllstände } \\
\text { zu symbolisieren. Zudem werden die Tätigkeiten der Mitarbeiter quittiert. }\end{array}$ \\
\hline
\end{tabular}




\subsection{Werkzeuginstandsetzung bei Maschinenausfällen}

Im Rahmen des ersten Anwendungsfalls liegt der Fokus auf der Kommunikationsverbesserung von am Prozess beteiligten Produktionsgruppen. Der im Folgenden vorgestellte Anwendungsfall wurde realweltlich in einer Stanzerei mit angegliedertem Werkzeugbau mit ungefähr 50 Mitarbeitern evaluiert. Die Produktionsmitarbeiter teilen sich auf die Bereiche Werkzeugbau inklusive Instandhaltung, Logistik und Produktion auf. Die Produktionsbereiche sind physisch auf zwei Gebäudekomplexe verteilt. Im Hauptgebäude befindet sich der Werkzeugbau mit Instandhaltungsabteilung und Verwaltung. Komplementiert wird dieses mit einer separaten Fertigungshalle, in der die Stanzmaschinen die Endprodukte fertigen. Aufgrund dieser Rahmenbedingungen ist es für das Unternehmen unerlässlich, eine möglichst lückenlose und umfassende Kommunikation zwischen den Produktionsmitarbeitern über die zwei Hallen sicherzustellen. Vor der Integration von smarten Geräten fand die Kommunikation über Telefonie oder direkte Gespräche statt, bei denen je nach Kontext des Gespräches, die Qualität der Informationsweitergabe nicht sichergestellt wurde. Der Hauptgrund für die Erfordernis einer kontinuierlichen direkten Kommunikation der Produktionsmitarbeiter sind Defekte von Produktionsmaschinen in der Fertigungshalle, die ein Produktionsmitarbeiter des Werkzeugbaus beheben oder bewerten muss. Ein spezieller Fall, der im Fokus der Analyse im Forschungsprojekt stand, ist ein Werkzeugbruch an einer der Stanzmaschinen, der dem Ablauf aus Abbildung 3 folgt.

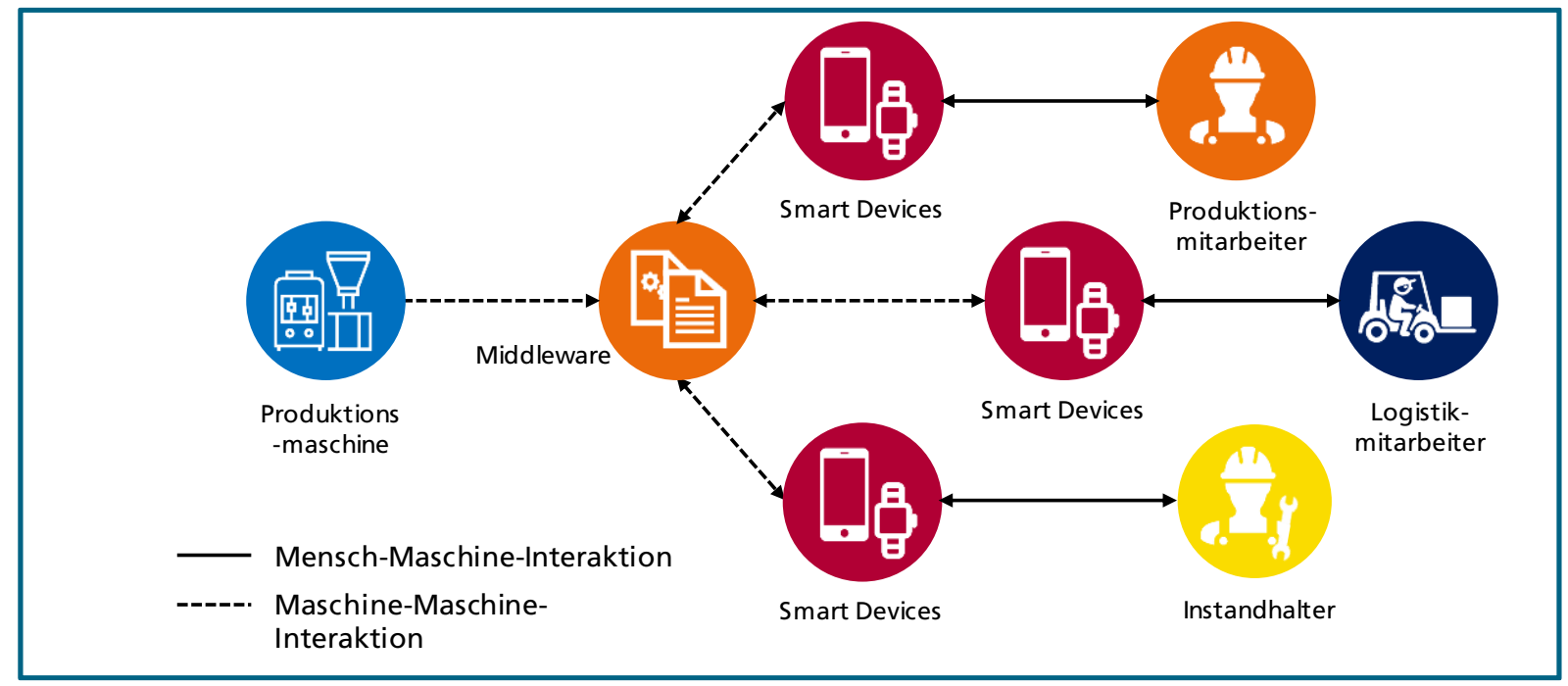

Abbildung 3: Schematische Darstellung von Anwendungsfall 1

Ein Produktionsmitarbeiter erfasst einen Werkzeugbruch an einer Stanzmaschine und evaluiert, ob er diesen beheben kann. Sofern der Produktionsmitarbeiter die Entscheidung trifft, dass eine Reparatur durch den Werkzeugbau notwendig ist, entfernt er das Werkzeug und erstellt einen Trigger über die Client-Applikation, um automatisiert einen Transportauftrag für die Logistik und einen Auftrag für den Werkzeugbau zu erstellen. Als smarte Geräte eigneten sich laut der Vorauswahl ein Smartphone oder Tablet, da der Produktionsmitarbeiter beim Melden des Werkzeugbruchs in der Lage sein muss, verschiedene Informationen zur weiteren Verarbeitung einzugeben. Dazu zählen Standortangaben, Fehlerart sowie Bezeichnung des defekten Werkzeugs, Zeit bis das Werkzeug transportiert werden kann und ggf. Bildmaterial, das ebenfalls für die Nutzung eines smarten Geräts mit Kamera spricht. Nach dem Absenden erhält der Logistiker einen Transportauftrag mit den relevanten Informationen, Zeitpunkt, Standort und Ziel. Im Rahmen der ersten Implementierung erhielt der Logistikmitarbeiter auf eigenen Wunsch ebenfalls ein Smartphone, dennoch war während der ersten Testphase die Ablenkung des Logistikers, der den größten Teil der Arbeitszeit einen Gabelstapler bedient, zu groß. Daher entschied man sich im weiteren Verlauf für die Nutzung einer smarten Uhr, die über ein haptisches Signal den Logistikmitarbeiter über einen neuen Auftrag informiert. Nach der Bestätigung durch den Logistikmitarbeiter erhält der Produktionsmitarbeiter des Werkzeugbaus eine Auftragserinnerung, sodass dieser die Wartung vorbereiten und das Werkzeug in Empfang nehmen kann. Die erneute Erinnerung des Produktionsmitarbeiters im Werkzeugbau ist eine erhebliche Verbesserung im Vergleich zum bisherigen Ablauf, da teilweise instand zusetzende Werkzeuge nach der Anlieferung gar nicht oder 
zu spät bemerkt wurden. Zum Abschluss des Anwendungsfalls gibt der Produktionsmitarbeiter aus dem Werkzeugbau die restliche Bearbeitungszeit über ein Smartphone oder Tablett ein, sobald er diese abschätzen kann. Dadurch wird der Rücktransport getriggert und der Logistikmitarbeiter erhält, analog zur Anlieferung, einen Transportauftrag und der Produktionsmitarbeiter den Zeitpunkt der Rücklieferung.

Neben der Notifikation neuer Nachrichten über die smarten Geräte war ein essentieller Punkt bei der Rückmeldung der Mitarbeiter der lückenlose und zeitnahe Versand der Nachrichten. Aufgrund der Weitläufigkeit des Geländes brach in den ersten Testphasen die Netzwerkverbindung der smarten Geräte regelmäßig ab, sodass die Nachrichten lediglich zeitverzögert übermittelt wurden. Eine weitere Rückmeldung war zudem, dass Notifikationen, nur schwer bemerkbar waren, da die Lautstärke der Smartphones für den Produktionsbereich und deren Geräuschkulisse ungenügend war. Ferner bemerkten die Produktionsmitarbeiter haptische Signale aufgrund der Sicherheitskleidung nicht. Diese Rückmeldungen konnten durch die Verwendung von Fitnessarmbändern am Handgelenk adressiert werden. Der Vorteil der Nutzung von Fitnessarmbändern lag dabei bei ihrem geringen Gewicht und ihrer, im Vergleich zu smarten Uhren, schmaleren Bauweise, sodass der Arbeitsschutz nicht beeinträchtigt wurde. Die Produktionsmitarbeiter bestätigten eine Erleichterung inklusive Qualitätssteigerungen in der Kommunikation und Zeitersparnisse bei der Werkzeugreparatur. Zudem wurde durch die Reduktion von nicht wertschöpfenden Tätigkeiten die Bearbeitungszeit eines Werkzeugbruches reduziert.

\subsection{Dokumentation von Maschinenstillständen und Fehlererfassung}

Ziel des zweiten Anwendungsfalls ist, analog zum vorherigen, die Kommunikationsverbesserung und Datenqualitätssteigerung bei der Dokumentation von Maschinenstillständen. Dieser Anwendungsfall konnte in einem metallverarbeitenden Unternehmen mit ca. 150 Mitarbeitern erprobt werden. Die Produktion dieses Unternehmen gliedert sich in die Bereiche Stanzumformtechnik, Biegetechnik und Fertigung von Hybridenbaugruppen. Im Fokus des Anwendungsfalls stand die Stanzumformtechnik mit über 20 Produktionsmaschinen, die an ein zentrales BDE-System zur Dokumentation des Maschinenzustandes angebunden sind. Das BDE-System erhält über die Produktionsmaschinen nahezu in Echtzeit Änderungen der Zustände und dokumentiert diese. Im Falle eines Stillstands verlangt das System die Eingabe eines Stillstandgrundes über ein BDE-Terminal, welches zentral in der Produktionshalle steht.

Vor der Umsetzung des Anwendungsfalles erhielten Produktionsmitarbeiter, die in einer Schicht für mehrere Produktionsmaschinen zuständig sind, über ein Lichtsignal an der Produktionsmaschine selbst die Maschinenstillstandinformation. Folglich war es Aufgabe des Produktionsmitarbeiters, kontinuierlich die Signalanlagen der Produktionsmaschinen zu überwachen. Dies erfolgte zusätzlich zu Haupttätigkeiten wie Instandhaltung und Rüsten. Des Weiteren lag es in der Verantwortung des Produktionsmitarbeiters, nach einem Stillstand den Grund im BDETerminal zu dokumentieren, wodurch Laufwege entstanden.

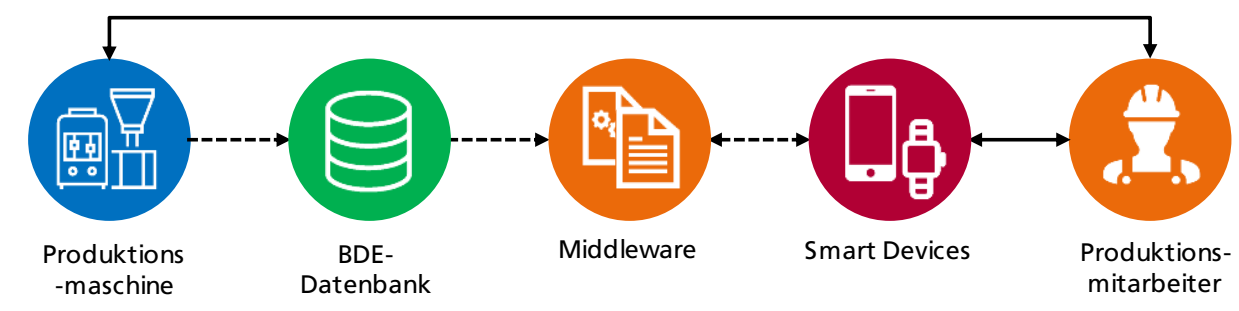

Mensch-Maschine-Interaktion

Maschine-Maschine-

Interaktion

Abbildung 4: Schematische Darstellung von Anwendungsfall 2 
Zur Minimierung der Laufwege war es daher üblich, Stillstände zu definierten Zeitpunkten, bspw. kurz vor Schichtende, gesammelt zu quittieren. Dieses Vorgehen war fehleranfällig, da pro Schicht eine zweitstellige Anzahl an Stillständen nicht ungewöhnlich ist. Im Rahmen des Forschungsprojekts wurde die Middleware eingesetzt, um Zustandsänderungen der Produktionsmaschinen als Trigger zur Information der Produktionsmitarbeiter zu verwenden (siehe Abbildung 4). Dadurch erhalten Produktionsmitarbeiter über ein Smartphone direkt die Information über einen Stillstand. Zudem ist es durch Smartphones möglich, direkt nach Behebung eines Stillstandes die Dokumentation anzuschließen. Sobald der Produktionsmitarbeiter eine Nachricht über einen Stillstand erhält, kann er den Empfang bestätigen. Anschließend erhält er den Auftrag den Stillstand zu beheben und direkt zu dokumentieren, sodass sich eine Reduktion der Laufwege ergibt. Im Verlauf des Forschungsvorhabens war es nach Rückmeldung der Produktionsmitarbeiter möglich, eine selbstorganisierte Aufgabenverteilung zu integrieren. Falls ein Produktionsmitarbeiter den Empfang nicht bestätigen kann, wird der Empfängerkreis automatisch erweitert.

Analog zum vorherigen Anwendungsfall bemängelten Produktionsmitarbeiter eine unzureichende Notifikationslautstärke. Zudem erhielt jeder Produktionsmitarbeiter während der ersten Testphase Stillstandnotifikationen aller Produktionsmaschinen ungeachtet dessen, dass ein Produktionsmitarbeiter lediglich für einen bestimmten Teil zuständig ist. Diese Rückmeldung wurde durch eine entsprechende Konfiguration adressiert. Eine Besonderheit in diesem Anwendungsfall war zudem die Identifikation von fehlerhaften Maschinenbenennungen innerhalb des BDE-Systems, sodass durch die Testphase die Datenqualität bei der Eingabe von Stillstandgründen sowie die allgemeine Datenqualität gesteigert werden konnte. Durch die Demonstratoren konnten im Unternehmen eine Reduktion von Laufwegen einhergehend mit einer Steigerung der Mitarbeiterzufriedenheit und der Zusage, die Smartphones ebenfalls zukünftig zu nutzen, festgestellt werden. Des Weiteren waren erste Verbesserungen der Datenqualität durch die direkte Eingabe von Stillstandgründen mittels Smartphones zu beobachten.

\subsection{Anleitung für Maschineneinstellungen}

Als Ergänzung zur Verbesserung der Kommunikations- und Datenqualität beinhaltet der dritte Anwendungsfall den Bereich der Wissensvermittlung und Sicherstellung von Standards bei der Bearbeitung von Routinetätigkeiten. Diese Aspekte sind für das dritte KMU mit ca. 85 Mitarbeitern in der Kunststoffspritzgussproduktion äußerst relevant. Kernherausforderungen dieses Unternehmens sind eine hohe Mitarbeiterfluktuation und teilweise Sprachbarrieren innerhalb der Belegschaft. Daher entstand der Anwendungsfall, Routinetätigkeiten so zu dokumentieren, dass ohne eine vergleichsweise lange Einarbeitungszeit neue oder unerfahrene Produktionsmitarbeiter die Tätigkeiten in einer vordefinierten Reihenfolge und Qualität abschließen können.

Vor Beginn des Forschungsprojekts erfolgte die Mitarbeiterschulung und Sicherstellung der Arbeitsqualität durch ein klassisches Ausbildungs- und Mehraugenprinzip. Eine Führungskraft, meist ein Meister oder Schichtleiter, bildet einen bisher unerfahrenen Produktionsmitarbeiter aus. Zusätzlich wird dem unerfahrenen Produktionsmitarbeiter ein erfahrener Kollege zur Seite gestellt, bis er selbstständig in der Lage ist, die geforderte Mindestqualität zu gewährleisten. Abschließend war es notwendig, dass eine Führungskraft nach Erledigung der Tätigkeiten die Ergebnisse abnimmt. Sind Produktionsmitarbeiter in ausreichender Menge geschult, besteht eine weitere Herausforderung bei Übergaben. Im Mehrschichtbetrieb muss eine Übergabe zwischen den Schichten etabliert und durchgeführt werden. Dennoch entstehen in der Realität bei der Übergabe Friktionen durch die Abwesenheit von Produktionsmitarbeitern oder eine ungenügende Informationsweitergabe.

Mit Hilfe von Smartphones und Tablets wurden diese Herausforderungen adressiert, indem für Routinetätigkeiten bisher papierbasierte Arbeitsablaufbeschreibungen digitalisiert wurden (siehe Abbildung 5). Insgesamt erstellte das Unternehmen sechs Arbeitsablaufbeschreibungen für die Rüstung, den Werkzeugwechsel an Spritzgussmaschinen und deren tägliche Reinigung im XLSX-Format. Produktionsmitarbeiter sind in der Lage über einen Baroder Quick-Response-Code (QR-Code) als Trigger, eine Arbeitsablaufbeschreibung zu starten. Im Verlauf der Testphasen entschieden sich die Produktionsmitarbeiter bevorzugt zur Nutzung eines Tablets, da dieses übersichtlich die teils langen Texte anzeigen konnte. In der Client-Applikation ist es möglich, dem Produktionsmitarbeiter die Reihenfolge der Tätigkeiten vorzuschlagen und, durch die Möglichkeit, erledigte Tätigkeiten zu markieren, den Fortschritt anzuzeigen. Ferner wurden einzelne Schritte durch Bildmaterial visualisiert, sodass Besonderheiten 
und kritische Handlungen hervorgehoben werden. Zusätzlich ist es möglich, den Status einer gestarteten Ablaufbeschreibung solange digital vorzuhalten, bis der vorgesehene Ablauf vollständig beendet ist. Produktionsmitarbeiter aus der folgenden Schicht konnten so bereits begonnene Ablaufbeschreibungen importieren und den Ablauf fortsetzen. Nach Beenden einer Tätigkeit gemäß der Ablaufbeschreibung werden Startzeitpunkt, Bezeichnung der Produktionsmaschine, Endzeitpunkt und alle erledigten Schritte gespeichert. Damit wird den Anforderungen zu Dokumentationspflichten für Kunden und Auditoren nachgekommen.

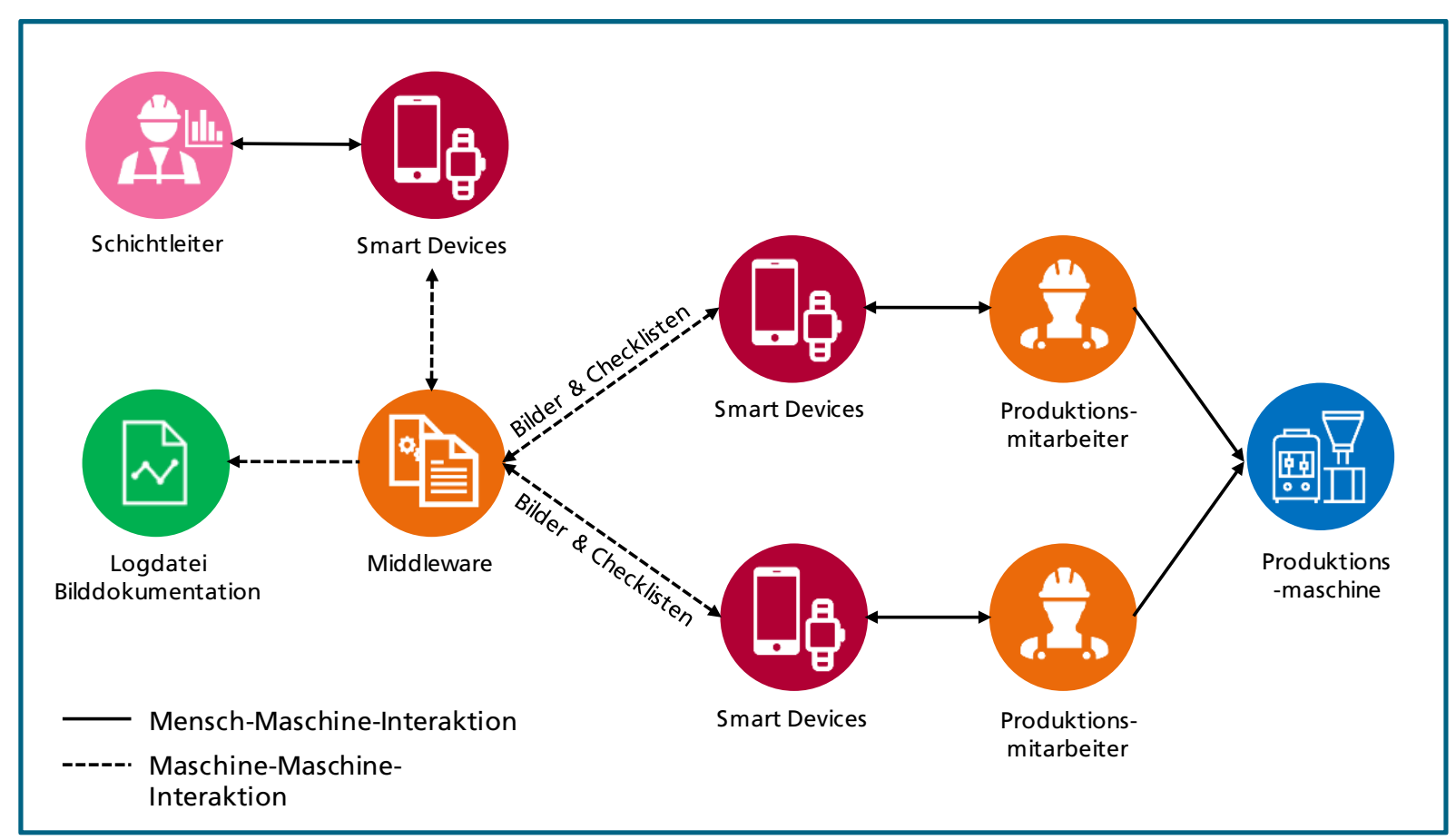

Abbildung 5: Schematische Darstellung von Anwendungsfall 3

Während der Testphasen gaben die Produktionsmitarbeiter positive Rückmeldungen zur Benutzung der digitalen Ablaufbeschreibungen, sodass die Funktionalitäten erweitert wurden. Als Erweiterung erhielt der Schichtleiter ein Smartphone, auf dem eine Übersicht der aktuell gestarteten Ablaufbeschreibungen ersichtlich war. Abschließend ließen sich folgende Verbesserungen feststellen: Reduktion von Schulungsaufwand, Erhöhung der Arbeitsqualität, hohe Akzeptanz der Tablets, Einsparung von Lagerflächen durch papierlose Dokumentation und intuitives Design der Benutzeroberfläche der Client-Applikation, sowie nachvollziehbare und unveränderbare Dokumentation der Arbeitsergebnisse.

\section{Allgemeine Erkenntnisse und Handlungsempfehlungen}

Nach der Implementierung der acht Anwendungsfälle war es mit dem gesamten Konsortium möglich, allgemeine Handlungsempfehlungen für die Integration von smarten Geräten in die Produktion von KMU abzuleiten.

\section{Empfehlung 1: Anwendungsfälle sind mit Beteiligten mehrerer Hierarchiestufen iterativ zu implementieren.}

Wie aus allen Anwendungsfällen ersichtlich, fungieren die Produktionsmitarbeiter als Nutzer der smarten Geräte. Deswegen zeigen die Erfahrungen, dass alle Beteiligten der Nutzung smarter Geräte offener gegenüberstehen, wenn sie frühzeitig in Diskussionen und insbesondere in die Auswahl und Definition der Anwendungsfälle eingebunden werden. Produktionsmitarbeiter kennen die einzelnen Produktionsschritte aus der täglichen Arbeit sehr gut - und daher meist auch Gründe für Ineffizienzen oder Fehler. Dieses Wissen hilft bei der Konfiguration der Middleware und Client-Applikation. Des Weiteren erhöht eine frühe Integration der Produktionsmitarbeiter deren Akzeptanz sowie Zufriedenheit. Daher sollten über ein gesamtes Projekt hinweg Mitarbeiter mehrerer Hierarchiestufen kontinuierlich zusammenarbeiten. Dies wird durch ein agiles Vorgehen unterstützt, in dem Produktionsmit- 
arbeiter die Demonstratoren und Nutzbarkeit der smarten Geräte bewerten können. Bereits in der nächsten Testphase sind die gewünschten Änderungen bewertbar, und die Produktionsmitarbeiter erkennen ihre Integration und Relevanz für das Projekt.

\section{Empfehlung 2: Für eine erfolgreiche Integration muss der Kontext der Implementierung analysiert werden.}

In nahezu allen Testphasen wurde als zentrale Rückmeldung die unzureichende Lautstärke oder zu schwache haptische Notifikationsweitergabe der smarten Geräte genannt. Dieser Umstand wirkte sich teils negativ auf die Mitarbeiterakzeptanz aus. Produktionsmitarbeiter merkten an, dass keine Prozessverbesserungen möglich sind, wenn smarte Geräte aufgrund der Umgebungsgeräusche nicht bemerkbar sind. Daher ist es essentiell, derartige Kontextfaktoren von zu Beginn an zu berücksichtigen. Im Forschungsprojekt konnte durch den Einsatz von smarten Uhren oder Armbändern in Kombination mit Tablets oder Smartphones sehr gut auf diese Kontextfaktoren reagiert werden. Des Weiteren ist es nach entsprechender Rüstung möglich, handelsübliche Geräte in der Produktion nachhaltig und langfristig einzusetzen. Während des gesamten Forschungsprojekts wurde kein smartes Gerät unbrauchbar, lediglich einige Schutzmechanismen mussten nach Beschädigungen ausgetauscht werden.

\section{Empfehlung 3: Infrastruktur und interdisziplinäre Projektteams führen zum Erfolg.}

Bei der Integration der Demonstratoren wurde insbesondere bei Anwendungspartnern mit mehreren Gebäuden oder größeren Produktionshallen eine schlechte Netzwerkkonnektivität zurückgemeldet. Meist wurden zu Beginn lediglich einzelne Netzwerkkomponenten für ein drahtloses Netzwerk installiert. Dies führte dazu, dass die ClientApplikation bei Verlust der Netzwerkverbindung keine Nachrichten mehr erhielt und zu einem späteren Zeitpunkt gepufferte und teilweise nicht mehr relevante Nachrichten versendet wurden. Daraus folgt die Empfehlung, bei der Einführung von smarten Geräten die Netzwerkinfrastruktur entsprechend auszulegen. Dies ist zudem ein gutes Beispiel für die produktive Zusammenarbeit eines interdisziplinären Teams, da zunächst von einem Implementierungsfehler im Demonstrator ausgegangen wurde. Erst durch die Diskussion zwischen Produktionsmitarbeitern, Projektverantwortlichen, IT-Abteilung sowie Entwicklern und Ingenieuren konnte der die tatsächliche Fehlerursache identifiziert und adressiert werden.

\section{Fazit}

Für die niedrigschwellige Einführung von IoT in KMU wurden anhand realer Anwendungsfälle Handlungsempfehlungen herausgearbeitet. Inhalt der Anwendungsfälle war die Analyse, wie verschiedene Klassen smarter Geräte in Produktionsprozessen von KMU sinnvoll eingesetzt werden können. Hierfür wurde ein Demonstrator bestehend aus einer Middleware und einer Client-Applikation implementiert. Die Funktionsweise des Demonstrators wurde für jeden Anwendungsfall mehrfach unter realen Produktionsbedingungen getestet und schrittweise verbessert. In allen Anwendungsfällen hat sich gezeigt, dass sich positive Effekte hinsichtlich Prozessqualität und -stabilität sowie Zeit- und Kosteneinsparungen erzielen lassen. Aufgrund der einfachen Integrierbarkeit der Demonstratoren und der Nutzung alltäglicher smarter Geräte können wir insbesondere KMU eine nützliche Lösung anbieten. Die modular aufgebaute Middleware stellt allgemeine Schnittstellen zu anderen Anwendungssystemen und Produktionsmaschinen bereit, sodass bisher verwendete Anwendungssysteme weiterverwendet werden können und keine tiefe Integration erforderlich ist. Produktionsmitarbeiter sind in der Lage, das System nach einer kurzen Schulung zu nutzen, da sie die Nutzung von smarten Geräten im privaten Umfeld gewohnt sind. Die Ergebnisse stellen eine gute Startlösung für KMU dar.

Analog zu jeder Forschung ist dieser Beitrag nicht frei von Limitationen. Es sei darauf hingewiesen, dass die Ergebnisse und daraus abgeleiteten Handlungsempfehlung auf vier Anwendungskontexten basieren, die entweder aus der metall- oder kunststoffverarbeitenden Industrie stammen. Zudem sind im Rahmen des Forschungsprojekts zwei Demonstratoren entstanden. Während der Evaluierung bestätigten die Produktionsmitarbeiter deren Nützlichkeit, allerdings muss für eine produktionsreife Nutzung der Demonstratoren weiter investiert werden. Des Weiteren wurden während der Evaluierung ein konkreter Anwendungsfall lediglich bei einem Anwendungspartner evaluiert, weshalb die Generalisierbarkeit eingeschränkt ist. Es ist sinnvoll, in einem nächsten Schritt die Anwendungsfälle bei weiteren Unternehmen zu evaluieren. 
Da es sich bei den entwickelten Demonstratoren um eine Startlösung handelt, können diese künftig aufgrund der modularen Architektur weiterentwickelt werden. Neben der Adressierung der Limitationen merkten die Produktionsmitarbeiter an, dass es wünschenswert wäre, über die smarten Geräte nicht nur lesend auf Produktionsmaschinen zugreifen zu können, sondern ebenfalls steuernd. Dieser Wunsch ist nachvollziehbar, da hierdurch eine weitere Reduktion von Laufwegen realisierbar wäre. Allerdings bringt dies einen weiteren Sicherheitsaspekt mit sich. Es muss sichergestellt sein, dass lediglich autorisierte Mitarbeiter steuernd eingreifen können und Angriffe von extern ausgeschlossen sind. Neben diesen Ausblicken aus den Rückmeldungen seien aktuelle Entwicklungstrends wie Augmented Reality und Predictive Maintenance genannt, die künftig bearbeitet werden sollen.

\section{Danksagung}

Die Autoren bedanken sich herzlich bei der Bayerischen Forschungsstiftung für die finanzielle Unterstützung als Fördergeber und dem gesamten Konsortium des Forschungsprojekts, ,SmarDes@Work-Smart Devices in der Produktion ". Dank den Anwendungspartnern BKW Kunststoff GmbH, biTTner Werkzeugbau GmbH, Dietz GmbH und REHAU AG +Co. KG war es jederzeit möglich, Erfahrungen und Einblicke aus der Industrie in das Projekt einzubringen. Die Entwicklungspartner camLine GmbH und Vogler Engineering $\mathrm{GmbH}$ realisierten die SoftwareDemonstratoren. Weitere Informationen zum Forschungsprojekt finden sich unter http://smart-devices.fim-rc.de.

\section{Literatur}

Aier S, Schelp J (2008) EAI und SOA-Was bleibt nach dem Hype? Multikonferenz Wirtschaftsinformatik, München 2008

Albayrak CA, Gadatsch A (2018) Sind kleinere und mittlere Unternehmen (KMU) bereits auf die Digitale Transformation vorbereitet? Multikonferenz Wirtschaftsinformatik, Lüneburg 2018

Arbeitskreis Industrie 4.0 (2013) Umsetzungsempfehlungen für das Zukunftsprojekt Industrie 4.0. https://www.bmbf.de/files/Umsetzungsempfehlungen_Industrie4_0.pdf. Zugegriffen: 19. Juni 2019

Bertschek I, Clement R, Buhr D, Hirsch-Kreinsen H, Falck O, Heimisch A, Jacob-Puchalska A, Mazat A (2015) Industrie 4.0: Digitale Wirtschaft Herausforderung und Chance für Unternehmen und Arbeitswelt. ifo Schnelldienst 68: S. 3-18

BMW Group (2015) New perspectives in production: BMW Group draws on digitalization to advance production network. https://www.press.bmwgroup.com/global/article/detail/T0229627EN/new-perspectives-in-production:-bmw-group-draws-on-digitalization-to-advance-production-network. Zugegriffen: 24. Juni 2019

Fleisch E, Weinberger M, Wortmann F (2015) Geschäftsmodelle im Internet der Dinge. Schmalenbachs Zeitschrift für betriebswirtschaftliche Forschung 67: S. 444-465.

Fraunhofer IML (2019) Smart Devices; Smart Devices versorgen Ihre Mitarbeiter zur richtigen Zeit am richtigen Ort mit den richtigen Informationen. https://www.iml.fraunhofer.de/de/themengebiete/informationslogistik_und_assistenzsysteme/smart_devices.html. Zugegriffen: 19. Juni 2019

Hausladen I (2010) Materialwirtschaft und Produktion heute. HMD - Praxis der Wirtschaftsinformatik 47: S. 6-16.

Kagermann H, Lukas W-D, Wahlster W (2011) Industrie 4.0: Mit dem Internet der Dinge auf dem Weg zur 4. industriellen Revolution. VDI nachrichten

Leyh C, Bley K (2016) Digitalisierung: Chance oder Risiko für den deutschen Mittelstand? - Eine Studie ausgewählter Unternehmen. HMD - Praxis der Wirtschaftsinformatik 53: S. 29-41. 
Muccini H, Moghaddam MT (2018) IoT Architectural Styles. In: Cuesta CE, Garlan D, Pérez J (Hrsg) Software Architecture. Springer International Publishing, Cham, S 68-85

Oberländer AM, Röglinger M, Rosemann M, Kees A (2018) Conceptualizing business-to-thing interactions - A sociomaterial perspective on the Internet of Things. European Journal of Information Systems 27: S. 486-502.

Österle H, Otto B (2010) Consortium Research. Business Information Systems Engineering 2: S. $283-293$.

Porter ME, Heppelmann JE (2014) How smart, connected products are transforming competition. Harvard business review 92: S. 64-88

Püschel L, Röglinger M, Schlott H (2017) Smart Things im Internet der Dinge — ein Klassifikationsansatz. Wirtschaftsinformatik und Management 9: S. 54-61.

Rüßmann M, Lorenz M, Gerbert P, Waldner M, Justus J, Engel P, Harnisch M (2015) Industry 4.0: The future of productivity and growth in manufacturing industries. Boston Consulting Group 9: S. 54-89

Statistisches Bundesamt (2019) Kleine und mittlere Unternehmen. https://www.destatis.de/DE/Themen/Branchen-Unternehmen/Unternehmen/Kleine-Unternehmen-Mittlere-Unternehmen/_inhalt.html. Zugegriffen: 19. Juni 2019

Virtual Reality Magazin (2015) Volkswagen: 3D-Datenbrille im Serieneinsatz. http://www.virtual-reality-magazin.de/volkswagen-3d-datenbrille-im-serieneinsatz. Zugegriffen: 24. Juni 2019

Winkelmann A, Klose K (2008) Experiences while selecting, adapting and implementing ERP systems in SMEs: a case study. AMCIS 2008 Proceedings: 257 